\title{
Molecularly targeted drugs for metastatic colorectal cancer
}

\author{
This article was published in the following Dove Press journal: \\ Drug Design, Development and Therapy \\ 31 October 2013 \\ Number of times this article has been viewed
}

\section{Ying-dong Cheng \\ Hua Yang \\ Guo-qing Chen \\ Zhi-cao Zhang}

Department of General Surgery, Xinqiao Hospital, Third Military

Medical University, Chongqing,

People's Republic of China
Correspondence: Hua Yang

Department of General Surgery, Xinqiao Hospital, Third Military Medical University, Xinqiao Street, Chongqing 400037, People's Republic of China

Tel +86236875 5705

Email hwbyang@yahoo.com
Abstract: The survival rate of patients with metastatic colorectal cancer (mCRC) has significantly improved with applications of molecularly targeted drugs, such as bevacizumab, and led to a substantial improvement in the overall survival rate. These drugs are capable of specifically targeting the inherent abnormal pathways in cancer cells, which are potentially less toxic than traditional nonselective chemotherapeutics. In this review, the recent clinical information about molecularly targeted therapy for $\mathrm{mCRC}$ is summarized, with specific focus on several of the US Food and Drug Administration-approved molecularly targeted drugs for the treatment of $\mathrm{mCRC}$ in the clinic. Progression-free and overall survival in patients with $\mathrm{mCRC}$ was improved greatly by the addition of bevacizumab and/or cetuximab to standard chemotherapy, in either first- or second-line treatment. Aflibercept has been used in combination with folinic acid (leucovorin)-fluorouracil-irinotecan (FOLFIRI) chemotherapy in mCRC patients and among patients with mCRC with wild-type $K R A S$, the outcomes were significantly improved by panitumumab in combination with folinic acid (leucovorin)-fluorouracil-oxaliplatin (FOLFOX) or FOLFIRI. Because of the new preliminary studies, it has been recommended that regorafenib be used with FOLFOX or FOLFIRI as first- or second-line treatment of mCRC chemotherapy. In summary, an era of new opportunities has been opened for treatment of $\mathrm{mCRC}$ and/or other malignancies, resulting from the discovery of new selective targeting drugs.

Keywords: metastatic colorectal cancer (mCRC), antiangiogenic drug, bevacizumab, aflibercept, regorafenib, cetuximab, panitumumab, clinical trial, molecularly targeted therapy

\section{Introduction}

Colorectal cancer (CRC) is one of the most malignant types of cancers. In the US and Europe, $\mathrm{CRC}$ is the second most frequent cancer that leads to death, which ranks below only lung cancer. ${ }^{1}$ The incidence of CRC in the People's Republic of China has been reported to increase annually and will continue to rise in the next few years. ${ }^{2}$ Currently, there are approximately 1.25 million patients diagnosed with CRC, and more than 600,000 patients will die from this disease every year. ${ }^{3}$ Metastases develop in at least $50 \%$ of CRC patients, and most of these patients have unresectable tumors. ${ }^{4}$ When tumor lesions are not fully resectable or become metastatic, the first treatment option is chemotherapy. Since the 1990s, fluorouracil (5FU)-based chemotherapy has improved the survival rate of patients with metastatic CRC (mCRC) to an overall survival (OS) of 12 months, and the addition of oxaliplatin and irinotecan increased the OS to approximately 18 months. ${ }^{5-8}$ The addition of molecularly targeted drugs, such as bevacizumab, led to a substantial jump in OS, which approached 30 months in some studies. $^{9}$ In 2007 , monoclonal antibody (mAb) therapy was first 
recommended for mCRC. ${ }^{10,11}$ According to the European and US guidelines, the combination of chemotherapy and a mAb is recommended for the first-line treatment of $\mathrm{mCRC}$, and the second-line treatment depends on the first-line regimen used. For patients with chemoresistant mCRC with wildtype KRAS, monotherapy with cetuximab or panitumumab is recommended. ${ }^{12}$ In addition, regorafenib was recently approved by the US Food and Drug Administration (FDA) for the treatment of $\mathrm{mCRC}$ patients who have been treated with chemotherapy previously, which is used in combination with an anti-vascular endothelial growth factor (VEGF) therapy, or with an anti-epidermal growth factor receptor (EGFR) therapy in case of wild-type KRAS. ${ }^{13}$ In recent years, significant advances have been made in the integration of targeted therapies in the treatment of $\mathrm{mCRC}$ and a plethora of new data have been published shedding light on the efficacy of targeted drugs in treatment of mCRC. However, these data have sometimes been inconsistent, resulting in a challenging environment in which physicians are required to make treatment choices. This review focuses on several FDA-approved molecularly targeted drugs that are being used regularly in the treatment of $\mathrm{mCRC}$.

\section{Bevacizumab, an angiogenesis inhibitor}

Bevacizumab (Avastin; Genentech/Roche, San Francisco, $\mathrm{CA}, \mathrm{USA}$ ) is a humanized $\mathrm{mAb}$ that inhibits the growth of new blood vessels. As the first clinically available inhibitor of angiogenesis in the US, bevacizumab has been licensed to treat various cancers, including breast (outside the US), ${ }^{14-16}$ glioblastoma (US only), ${ }^{17,18}$ lung, ${ }^{19,20}$ kidney, ${ }^{21,22}$ ovarian $^{23-25}$ and CRC. ${ }^{26-28}$ Mechanically, bevacizumab inhibits VEGF-A, ${ }^{29}$ a chemical signal that mediates angiogenesis, which is required for the development of cancer. ${ }^{30,31}$ Figure 1 shows how the VEGF-A signaling pathway is linked to its main biological functions. VEGF-A can bind VEGF receptor (VEGFR)-2 dimer. Neuropilin (NRP)-1 and -2 are co-receptors that stabilize the VEGFR-2 dimer. ${ }^{32}$ Upon ligand binding to VEGFR-2 dimer, several signaling pathways can be activated, affecting diverse biological processes in endothelial and cancer cells. Anti-VEGF-A mAb, such as bevacizumab, can bind VEGF-A and block its function. While it has been clearly demonstrated that bevacizumab has antitumor efficacy in various cancers, especially in combination with conventional chemotherapy, its exact mechanism of action remains not fully understood. Continued VEGF-A inhibition with bevacizumab can play an important role in improving the overall success of therapy for patients who have mCRC. ${ }^{9}$ However, many patients inevitably relapsed due to the newly acquired resistance, even though the progression-free survival (PFS) was statistically increased. ${ }^{33,34}$

When combined with 5FU-based chemotherapy, bevacizumab was found to significantly prolong the OS and PFS of patients with mCRC. For this reason, bevacizumab was first approved in 2004 for the combined use with standard chemotherapy for the treatment of mCRC. ${ }^{35,36}$ In 2005, the European Medicines Agency also approved bevacizumab in combination with irinotecan, a chemotherapeutic drug that prevents DNA from unwinding by inhibition of topoisomerase 1 for first-line treatment of mCRC. ${ }^{37}$ Bevacizumab was further approved for $\mathrm{mCRC}$ in combination with standard fluoropyrimidine-based chemotherapy after its benefits were demonstrated by randomized studies. ${ }^{38,39}$ More recently, on January 23, 2013, bevacizumab was approved to be used in combination with fluoropyrimidine-based irinotecan or oxaliplatin chemotherapy for the treatment of mCRC. ${ }^{40}$ The approval allows patients who received the first-line treatment with bevacizumab plus an irinotecan- or oxaliplatin-containing chemotherapy to continue to receive bevacizumab plus a different irinotecan- or oxaliplatincontaining chemotherapy as the second-line treatment after their cancer worsens. ${ }^{40}$

The use of bevacizumab with standard chemotherapy has improved PFS and OS for the treatment of $\mathrm{mCRC}$ in both first- and second-line treatment; however, the clinical significance of maintenance bevacizumab remains controversial. In a Phase III trial to compare the efficacy and safety of bevacizumab alone with bevacizumab and capecitabine plus oxaliplatin (XELOX) as maintenance treatment following induction chemotherapy with XELOX plus bevacizumab in the first-line treatment of patients with mCRC, Díaz-Rubio et al did not find statistically significant differences in the median PFS or OS times between bevacizumab-treated versus XELOX plus bevacizumab-treated patients. ${ }^{41}$ The results of a recent retrospective study indicate that the maintenance therapy with bevacizumab is a safe and valuable option in mCRC patients, especially in those who achieve an objective response after first-line chemotherapy. ${ }^{42}$ In addition, two reports from the 2013 American Society of Clinical Oncology (ASCO) annual meeting support the use of bevacizumab in maintenance treatment of mCRC. ${ }^{43,44}$

In summary, PFS and OS have been significantly improved by the addition of bevacizumab to standard chemotherapy in patients with $\mathrm{mCRC}$ in both first- and second-line treatment. Still, the role of bevacizumab as maintenance treatment of mCRC needs further studies. 


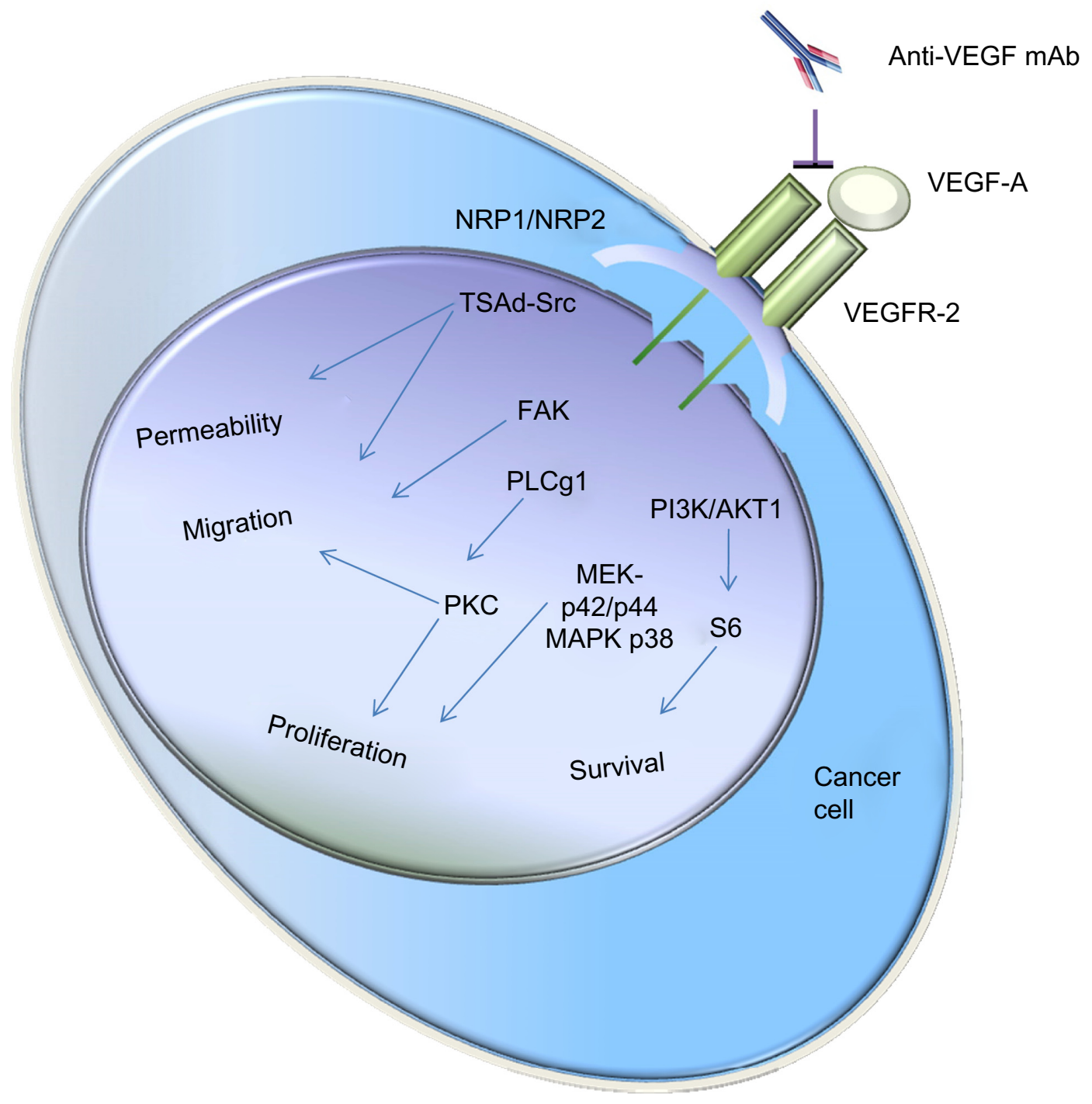

Figure I Schematic representation of how the VEGF-A signaling pathway is linked to its main biological functions.

Notes: VEGF-A can bind VEGFR-2 dimer. NRP-I and -2 are co-receptors that stabilize the VEGFR-2 dimer. Upon ligand binding to VEGFR-2 dimer, several signaling pathways can be activated, affecting diverse biological processes in endothelial and cancer cells. Anti-VEGF-A mAbs, such as bevacizumab or the fusion protein aflibercept, can bind to VEGF-A and block its function.

Abbreviations: mAb, monoclonal antibody; NRP, neuropilin; VEGF, vascular endothelial growth factor; VEGFR, VEGF receptor; PKC, protein kinase C; PLC, phospholipase C; FAK, focal adhesion protein; TSAd-Src, T cell-specific adaptor protein containing an Src homology.

\section{Aflibercept, a fusion protein}

Aflibercept (ZALTRAP; co-developed by Sanofi-Aventis and Regeneron Pharmaceuticals, Inc, Tarrytown, NY, USA) is a fusion protein for the treatment of wet macular degeneration ${ }^{45,46}$ and mCRC. ${ }^{47}$ The mechanism of aflibercept action is to target VEGF-A, VEGF-B, and placental growth factor (PIGF) by blocking angiogenesis. It also prevents the activation of VEGFR-1 and VEGFR-2 by these ligands. ${ }^{47,48}$ As a member of the VEGF family, PIGF specifically binds to VEGFR-1 and enhances VEGF-A expression, which plays an important role in angiogenesis. ${ }^{49}$ PIGF expression is also upregulated by anti-VEGF therapy in cancer patients, indicating that PIGF may also play a role in the resistance to
anti-VEGF treatment. ${ }^{50,51}$ In addition, aflibercept was shown to inhibit tumor proliferation, angiogenesis, and metastases in tumor-bearing mouse model. ${ }^{52,53}$

On August 3, 2012, aflibercept was approved by the FDA for patients who were previously treated with mCRC.47 The approval stipulates that aflibercept can be used in combination with folinic acid (leucovorin)-5FU-irinotecan (FOLFIRI) chemotherapy for mCRC patients whose cancer has progressed or demonstrated resistance to oxaliplatin (Eloxatin; Sanofi-Aventis)-based chemotherapy. The benefit of aflibercept in combination with FOLFIRI was confirmed in the Phase III VELOUR trial. ${ }^{54}$ In this study, aflibercept or placebo combined with FOLFIRI was administered to 
patients with mCRC at $4 \mathrm{mg} / \mathrm{kg}$ intravenously every 2 weeks. The results demonstrate that the aflibercept-containing group had better PFS (6.9 versus 4.67 months; hazard ratio [HR] 0.758; $P<0.0001)$ and OS (13.5 versus 12.06 months; HR $0.817 ; P=0.0032$ ) compared to the control group. In the aflibercept arm, the overall response rate was $19.8 \%$, whereas in the placebo arm, the overall response rate was $11.1 \%(P=0.0001) .^{54,55}$

It seems that the benefit achieved by aflibercept and bevacizumab are comparable in second-line settings. As shown in a clinical trial, a median OS improvement of 1.4 months (HR $0.81 ; 95 \%$ confidence interval $[\mathrm{CI}]: 0.69-0.94 ; P=0.0062$ ) was achieved when bevacizumab was continued in the second-line while switching the cytotoxic chemotherapy. ${ }^{56}$ Meanwhile, in the VELOUR trial, the addition of aflibercept to FOLFIRI resulted in a comparable median OS survival improvement of 1.44 months (HR 0.817; 95.34\% CI: $0.713-0.937 ; P=0.0032) .{ }^{54}$ However, compared to bevacizumab, aflibercept appeared to have a higher frequency of vascular-related adverse events. Therefore, aflibercept is not recommended for routine use in $\mathrm{mCRC}$ patients who progress on oxaliplatin-containing treatment.

\section{Cetuximab, a chimeric monoclonal antibody}

Cetuximab (Erbitux), a chimeric (mouse/human) monoclonal antibody, is manufactured and distributed by BristolMyers Squibb (New York, NY, USA) and Eli Lilly and Company (Indianapolis, IN, USA). This drug is specific to the EGFR, ${ }^{57,58}$ which is administered by intravenous infusion. ${ }^{59}$ Cetuximab is able to induce various proapoptotic factors, such as Bax, leading to the activation of caspases and thus triggering apoptosis. ${ }^{60,61}$ In addition, cetuximab can also recruit immune cells to tumor cells, thereby inducing antibody-dependent cellular cytotoxicity in vivo. ${ }^{62,63}$ Figure 2 shows the EGFR pathway and its main downstream effectors, PI3K/AKT and KRAS/BRAF/MEK/MERK. Activated AKT and MEK/MERK can induce cancer cell proliferation and invasion. In addition, activated AKT can induce cancer stem cell renewal and differentiation. ${ }^{64,65}$

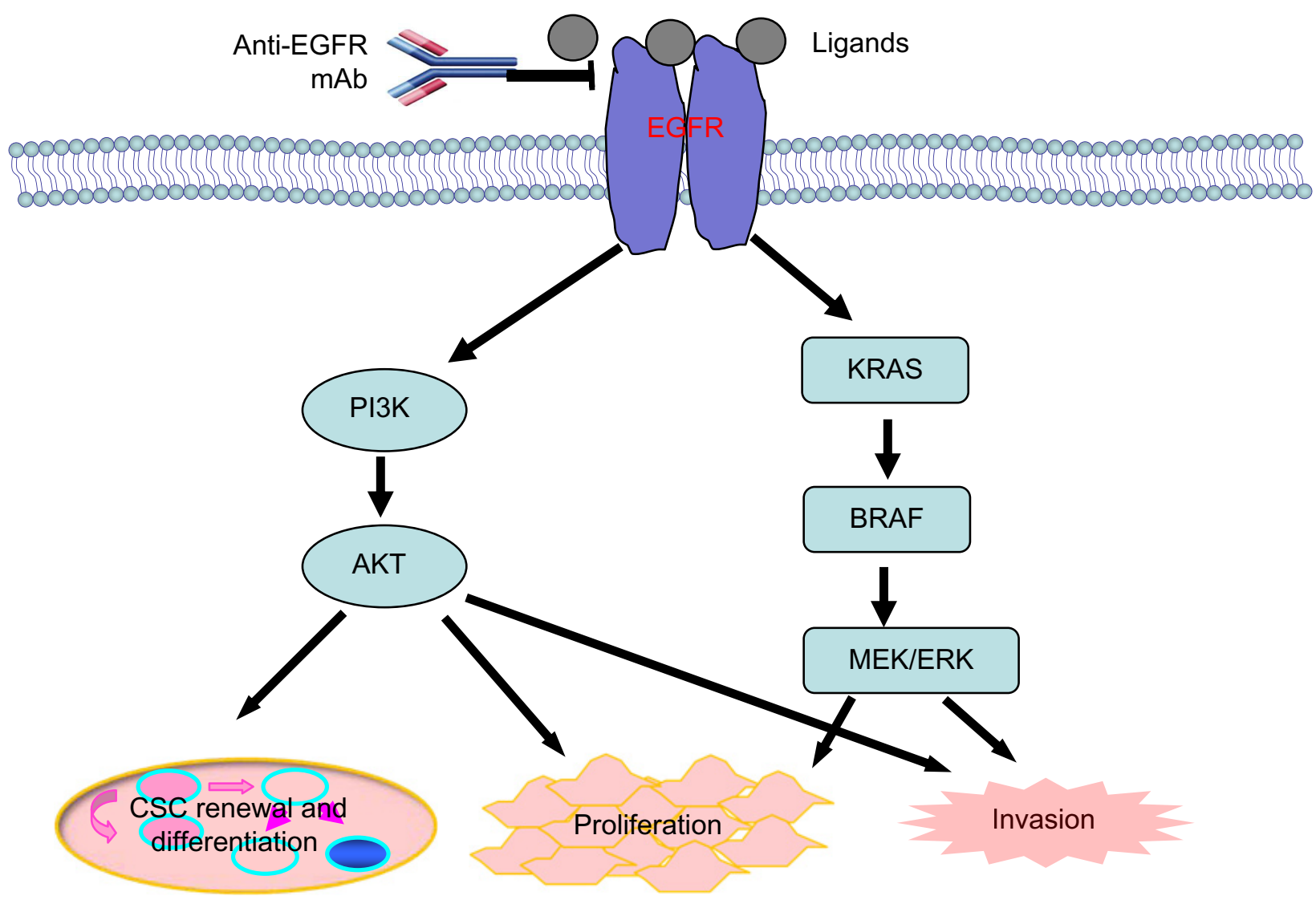

Figure 2 An overview of the EGFR pathway and its main downstream effectors, PI3K/AKT and KRAS/BRAF/MEK/MERK.

Notes: Activated AKT and MEK/MERK can induce cancer cell proliferation and invasion. In addition, activated AKT can induce cancer stem cell renewal and differentiation. Anti-EGFR mAbs, such as cetuximab or panitumumab, can bind EGFR and block its function.

Abbreviations: EGFR, epidermal growth factor receptor; mAb, monoclonal antibody; CSC, cancer stem cell. 
Anti-EGFR mAbs, such as cetuximab, can bind EGFR and block its function.

Initially, cetuximab was used in the palliative treatment of tumor. This drug, either as monotherapy or in combination with chemotherapy and/or radiation, particularly in the settings of $\mathrm{mCRC}$, has shown positive antitumor activity in clinical trials. ${ }^{66-69}$ In 2004, cetuximab was approved to be used for patients with EGFR-expressing mCRC who are refractory to irinotecan-based chemotherapy. ${ }^{70}$ Subsequently, there have been several clinical trials supporting the use of cetuximab for the treatment of mCRC. On July 6, 2012, the FDA approved the combination of cetuximab with FOLFIRI as the first-line treatment for patients with mutation-negative (wild-type) $K$-ras and EGFR-expressing mCRC. ${ }^{71-73}$ Concurrent with this approval, the therascreen $^{\circledR}$ KRAS RGQ PCR Kit (QIAGEN Manchester, Ltd) was also approved by the FDA for determining the K-ras mutations. The approval of cetuximab and KRAS mutation kit was based on the results of the CRYSTAL trial and two supportive studies, CA225025 and EMR 62 202-047 (OPUS), which made retrospective analyses of tumor samples from a large number of patients in accordance with $K$-ras mutation status. ${ }^{74,75}$ The addition of cetuximab to chemotherapy has significantly improved the OS, PFS, and the overall response rates in patients with $K$-ras wild-type tumors. However, no benefit, or even potential harm, has been observed in patients with $K$-ras mutant tumors.

The efficacy of down-staging programs in mCRC patients can be improved by the addition of cetuximab to conventional chemotherapy regimens, which may offer patients potential curative resection. However, it is well recognized that both intrinsic and acquired resistance have been developed despite the clinical gains arising from use of cetuximab. ${ }^{76}$ Therefore, there remains the need to develop more efficient antibodybased anti-EGFR therapies.

\section{Panitumumab, a fully human monoclonal antibody}

Panitumumab (Vectibix; Amgen Inc, Thousand Oaks, CA, USA) specifically blocks the EGFR extracellular domain. As a fully human monoclonal antibody, it can be used as a single drug in patients who are chemotherapy refractory or in different combinations. In either setting, panitumumab has been shown to be well tolerated and efficacious. ${ }^{77,78}$

Panitumumab was first approved in September 2006 in the US and in 2007 in Europe as a monotherapy for the treatment of mCRC that is EGFR-expressing and refractory to fluoropyrimidine-, oxaliplatin-, and irinotecan-based chemotherapy.
However, in patients with KRAS mutations in codons 12 or 13, use of panitumumab is not recommended. ${ }^{79,80}$ Recently, clinical studies have been conducted focusing on the potential benefits of treatment of $\mathrm{mCRC}$ with panitumumab in combination with chemotherapy. The results of these studies support that the clinical outcomes of patients with wild-type $K R A S$ are improved by the addition of panitumumab to chemotherapy. ${ }^{81-84}$ In another study, PFS was significantly improved by the addition of panitumumab to first-line folinic acid (leucovorin)-5FU-oxaliplatin (FOLFOX)4, which was 9.6 versus 8.0 months compared with FOLFOX4 alone (HR 0.80; 95\% CI: 0.66-0.97; $P=0.02$ ). However, addition of panitumumab to FOLFOX4 did not significantly improve the median OS in the patients with wild-type $K R A S$ tumors (23.9 versus 19.7 months compared to FOLFOX4 alone; HR $0.83 ; 95 \%$ CI: $0.67-1.02 ; P=0.072) .{ }^{81}$ In case of failure of initial treatment for $\mathrm{mCRC}$ with one prior chemotherapy regimen, the addition of panitumumab to FOLFIRI significantly improves the PFS in the wild-type KRAS subpopulation, with 5.9 months for panitumumab-FOLFIRI versus 3.9 months for FOLFIRI alone (HR 0.73; 95\% CI: 0.59-0.90; $P=0.004) .{ }^{82}$ A trend toward increased OS was also observed, although it was not statistically significant. The median OS was improved from 12.5 to 14.5 months, and the response rate increased from $10 \%$ to $35 \%$ with the addition of panitumumab compared to FOLFIRI alone. ${ }^{82}$ However, no difference in efficacy was observed in patients with mutant KRAS. ${ }^{82}$ Additional reports also demonstrate that, for patients with wild-type KRAS tumors, the objective response rate, PFS, and OS are numerically improved by panitumumab plus FOLFIRI. ${ }^{83,84}$

Overall, the results of the above studies support that panitumumab in combination with FOLFOX or FOLFIRI improves the outcomes among patients with $\mathrm{MCRC}$ with wild-type $K R A S$.

\section{Regorafenib, a multikinase inhibitor}

As an oral multikinase inhibitor, regorafenib ([BAY 73-4506] Stivarga; Bayer AG, Leverkusen, Germany) targets angiogenic, stromal, oncogenic receptor tyrosine kinase (RTK) as well as tumor microenvironment (platelet-derived growth factor receptor [PDGFR] and fibroblast growth factor receptor [FGFR]). ${ }^{85}$ Multiple membrane-bound and intracellular kinases are inhibited by regorafenib and its active metabolites. Therefore, multiple normal cellular functions and pathologic processes are inhibited, including the RET, VEGFR1, VEGFR2, VEGFR3, KIT, PDGFR-alpha, PDGFRbeta, FGFR1, FGFR2, TIE2, DDR2, Trk2A, Eph2A, RAF-1, BRAF, BRAF ${ }^{\mathrm{V} 600 \mathrm{E}}, \mathrm{SAPK} 2$, PTK5, and Abl pathways. ${ }^{85,86}$ It 
was further reported that regorafenib is effective in inhibiting angiogenesis, tumorigenesis, and metastasis in a highly aggressive metastatic colon cancer murine model, indicating its potential in the treatment of advanced CRCs. ${ }^{87}$

On September 27, 2012, the FDA approved regorafenib to be used in combination with an anti-VEGF therapy (or an anti-EGFR therapy, if wild-type KRAS), for the treatment of $\mathrm{mCRC}$ that has been treated with fluoropyrimidine-, oxaliplatin-, and irinotecan-based chemotherapy. ${ }^{13}$ In an international, randomized (2:1), double-blind, and placebo-controlled trial (study 14387) conducted in 760 patients with previously treated mCRC, regorafenib treatment resulted in a statistically significant prolongation in OS [HR 0.77; 95\% CI: 0.64-0.94; $P=0.0102$ ], with 6.4 months (95\% CI: 5.8-7.3) of median survival time in the regorafenib group versus 5.0 months (95\% CI: 4.4-5.8) in the placebo group. A statistically significant improvement in PFS was also demonstrated in patients who were treated with regorafenib [HR 0.49; 95\% CI: $0.42-0.58 ; P<0.0001]$. Specifically, the median PFS in patients receiving regorafenib was 2.0 months $(95 \%$ CI: 1.9-2.3), whereas, in the placebo group, the median PFS was 1.7 months (95\% CI: $1.7-1.8) .{ }^{13}$

As shown in a recent preliminary study, regorafenib in combination with FOLFOX or FOLFIRI has an acceptable tolerability as first- or second-line treatment of $\mathrm{mCRC}$ chemotherapy. ${ }^{88}$ In other tumor types, regorafenib has also shown exciting potential, especially in gastrointestinal stromal tumors. ${ }^{86,89}$

\section{Conclusion}

With the discovery of a plethora of molecular cellular targets, a large number of selective targeting drugs have been generated, and this has opened a new era for cancer therapy. These drugs specifically target the inherent abnormalities of cancer cells, which is potentially less toxic than traditional nonselective cytotoxic drugs. Addition of these drugs, such as bevacizumab and/or cetuximab, to standard chemotherapy has resulted in improved PFS and OS in patients with $\mathrm{mCRC}$, either in first- or second-line treatment. In patients with $\mathrm{mCRC}$ that has progressed on or is resistant to oxaliplatin (Eloxatin)-based chemotherapy, aflibercept has been used in combination with FOLFIRI chemotherapy. Among patients with mCRC with wild-type $K R A S$, the outcomes are improved when panitumumab is added to FOLFOX or FOLFIRI chemotherapy. Regorafenib can be used in combination with FOLFOX or FOLFIRI as first- or second-line treatment of $\mathrm{mCRC}$ chemotherapy.

\section{Disclosure}

The authors report no conflicts of interest in this work. The authors retained full editorial control over the content of the manuscript and received no compensation from any party for their work.

\section{References}

1. Jemal A, Center MM, DeSantis C, Ward EM. Global patterns of cancer incidence and mortality rates and trends. Cancer Epidemiol Biomarkers Prev. 2010;19(8):1893-1907.

2. Dai Z, Zheng RS, Zou XN, et al. [Analysis and prediction of colorectal cancer incidence trend in China]. Zhonghua Yu Fang Yi Xue Za Zhi. 2012;46:598-603. Chinese.

3. Globocan 2008 cancer fact sheet [webpage on the Internet]. Lyon: IARC; 2010. Available from: http://globocan.iarc.fr/factsheets/cancers/ colorectal.asp. Accessed August 15, 2013.

4. Van Cutsem E, Nordlinger B, Cervantes A; ESMO Guidelines Working Group. Advanced colorectal cancer: ESMO Clinical Practice Guidelines for treatment. Ann Oncol. 2010;21 Suppl 5:v93-v97.

5. André T, Louvet C, Maindrault-Goebel F, et al. CPT-11 (irinotecan) addition to bimonthly, high-dose leucovorin and bolus and continuousinfusion 5-fluorouracil (FOLFIRI) for pretreated metastatic colorectal cancer. GERCOR. Eur J Cancer. 1999;35:1343-1347.

6. Cheeseman SL, Joel SP, Chester JD, et al. A 'modified de Gramont' regimen of fluorouracil, alone and with oxaliplatin, for advanced colorectal cancer. Br J Cancer. 2002;87:393-399.

7. Goldberg RM, Sargent DJ, Morton RF, et al. A randomized controlled trial of fluorouracil plus leucovorin, irinotecan, and oxaliplatin combinations in patients with previously untreated metastatic colorectal cancer. J Clin Oncol. 2004;22:23-30.

8. Grothey A, Sargent D, Goldberg RM, Schmoll HJ. Survival of patients with advanced colorectal cancer improves with the availability of fluorouracil-leucovorin, irinotecan, and oxaliplatin in the course of treatment. J Clin Oncol. 2004;22:1209-1214.

9. Grothey A, Sugrue MM, Purdie DM, et al. Bevacizumab beyond first progression is associated with prolonged overall survival in metastatic colorectal cancer: results from a large observational cohort study (BRiTE). J Clin Oncol. 2008;26:5326-5334.

10. Scott LJ. Bevacizumab: in first-line treatment of metastatic breast cancer. Drugs. 2007;67:1793-1799.

11. Zhu Z. Targeted cancer therapies based on antibodies directed against epidermal growth factor receptor: status and perspectives. Acta Pharmacol Sin. 2007;28:1476-1493.

12. Edwards MS, Chadda SD, Zhao Z, Barber BL, Sykes DP. A systematic review of treatment guidelines for metastatic colorectal cancer. Colorectal Dis. 2012;14:e31-e47.

13. Regorafenib FAf. Previously Treated Metastatic Colorectal Cancer. http:// en.wikipedia.org/wiki/Regorafenib. Accessed October 15, 2013.

14. Stevenson CE, Nagahashi M, Ramachandran S, Yamada A, Bear HD, Takabe K. Bevacizumab and breast cancer: what does the future hold? Future Oncol. 2012;8:403-414.

15. Rodgers M, Soares M, Epstein D, Yang H, Fox D, Eastwood A. Bevacizumab in combination with a taxane for the first-line treatment of HER2-negative metastatic breast cancer. Health Technol Assess. 2011;15 Suppl 1:1-12.

16. Miles D, Zielinski C, Martin M, Vrdoljak E, Robert N. Combining capecitabine and bevacizumab in metastatic breast cancer: a comprehensive review. Eur J Cancer. 2012;48:482-491.

17. Narita Y. Drug review: safety and efficacy of bevacizumab for glioblastoma and other brain tumors. Jpn J Clin Oncol. 2013;43:587-595.

18. Rahmathulla G, Hovey EJ, Hashemi-Sadraei N, Ahluwalia MS. Bevacizumab in high-grade gliomas: a review of its uses, toxicity assessment, and future treatment challenges. Onco Targets Ther. 2013;6:371-389. 
19. Soria JC, Mauguen A, Reck M, et al; meta-analysis of bevacizumab in advanced NSCLC collaborative group. Systematic review and meta-analysis of randomised, phase II/III trials adding bevacizumab to platinum-based chemotherapy as first-line treatment in patients with advanced non-small-cell lung cancer. Ann Oncol. 2013;24:20-30.

20. Planchard D. Bevacizumab in non-small-cell lung cancer: a review. Expert Rev Anticancer Ther. 2011;11:1163-1179.

21. Melichar B, Procházková-Študentová H, Vitásková D. Bevacizumab in combination with IFN-alpha in metastatic renal cell carcinoma: the AVOREN trial. Expert Rev Anticancer Ther. 2012;12:1253-1261.

22. Di Lorenzo G, Porta C, Bellmunt J, et al. Toxicities of targeted therapy and their management in kidney cancer. Eur Urol. 2011;59:526-540.

23. Garcia A, Singh H. Bevacizumab and ovarian cancer. Ther Adv Med Oncol. 2013;5:133-141.

24. Miyake TM, Sood AK, Coleman RL. Contemporary use of bevacizumab in ovarian cancer. Expert Opin Biol Ther. 2013;13:283-294.

25. Eskander RN, Randall LM. Bevacizumab in the treatment of ovarian cancer. Biologics. 2011;5:1-5.

26. Yeung Y, Tebbutt NC. Bevacizumab in colorectal cancer: current and future directions. Expert Rev Anticancer Ther. 2012;12:1263-1273.

27. Whyte S, Pandor A, Stevenson M. Bevacizumab for metastatic colorectal cancer: a NICE single technology appraisal. Pharmacoeconomics. 2012;30:1119-1132.

28. Strickler JH, Hurwitz HI. Bevacizumab-based therapies in the firstline treatment of metastatic colorectal cancer. Oncologist. 2012;17: 513-524.

29. Grothey A, Galanis E. Targeting angiogenesis: progress with antiVEGF treatment with large molecules. Nat Rev Clin Oncol. 2009;6: 507-518.

30. Shibuya M. Vascular endothelial growth factor and its receptor system: physiological functions in angiogenesis and pathological roles in various diseases. J Biochem. 2013;153:13-19.

31. Giacca M, Zacchigna S. VEGF gene therapy: therapeutic angiogenesis in the clinic and beyond. Gene Ther. 2012;19:622-629.

32. Wang L, Zeng H, Wang P, Soker S, Mukhopadhyay D. Neuropilin-1mediated vascular permeability factor/vascular endothelial growth factor-dependent endothelial cell migration. J Biol Chem. 2003;278: 48848-48860.

33. Giuliano S, Pages G. Mechanisms of resistance to anti-angiogenesis therapies. Biochimie. 2013;95:1110-1119.

34. Moreno Garcia V, Basu B, Molife LR, Kaye SB. Combining antiangiogenics to overcome resistance: rationale and clinical experience. Clin Cancer Res. 2012;18:3750-3761.

35. Olszewski AJ, Grossbard ML, Kozuch PS. The horizon of antiangiogenic therapy for colorectal cancer. Oncology (Williston Park). 2005;19: 297-306; discussion 306, 308, 317-333.

36. Ellis LM. Bevacizumab. Nat Rev Drug Discov. 2005;Suppl:S8-S9.

37. Rougier P, Lepere C. Second-line treatment of patients with metastatic colorectal cancer. Semin Oncol. 2005;32:S48-S54.

38. Giantonio BJ, Catalano PJ, Meropol NJ, et al; Eastern Cooperative Oncology Group Study E3200. Bevacizumab in combination with oxaliplatin, fluorouracil, and leucovorin (FOLFOX4) for previously treated metastatic colorectal cancer: results from the Eastern Cooperative Oncology Group Study E3200. J Clin Oncol. 2007;25:1539-1544.

39. Saltz LB, Clarke S, Díaz-Rubio E, et al. Bevacizumab in combination with oxaliplatin-based chemotherapy as first-line therapy in metastatic colorectal cancer: a randomized phase III study. J Clin Oncol. 2008;26: 2013-2019.

40. FDA approval for bevacizumab [webpage on the Internet]. Bethesda, MD: National Cancer Institute. Available from: http://www.cancer.gov/ cancertopics/druginfo/fda-bevacizumab. Accessed August 15, 2013.

41. Díaz-Rubio E, Gómez-España A, Massutí B, et al; Spanish Cooperative Group for the Treatment of Digestive Tumors. First-line XELOX plus bevacizumab followed by XELOX plus bevacizumab or single-agent bevacizumab as maintenance therapy in patients with metastatic colorectal cancer: the phase III MACRO TTD study. Oncologist. 2012;17: $15-25$.
42. Moscetti L, Nelli F, Fabbri MA, et al. Maintenance single-agent bevacizumab or observation after first-line chemotherapy in patients with metastatic colorectal cancer: a multicenter retrospective study. Invest New Drugs. 2013;31(4):1035-1043.

43. Greil R, Von Moos R, Bennouna J, et al. Bevacizumab plus chemotherapy continued beyond first disease progression in patients with metastatic colorectal cancer previously treated with bevacizumabbased therapy: patterns of disease progression and outcomes based on extent of disease in the ML18147 study. 2013 ASCO Annual Meeting. J Clin Oncol. 2013;31(Suppl; abstr 3604).

44. Koopman M, Simkens LHJ, Ten Tije AJ, et al. Maintenance treatment with capecitabine and bevacizumab versus observation after induction treatment with chemotherapy and bevacizumab in metastatic colorectal cancer (mCRC): the phase III CAIRO3 study of the Dutch Colorectal Cancer Group (DCCG). 2013 ASCO Annual Meeting. J Clin Oncol. 2013;31(Suppl; abstr 3502).

45. Evoy KE, Abel SR. Aflibercept: newly approved for the treatment of macular edema following central retinal vein occlusion. Ann Pharmacother. 2013;47:819-827.

46. Thomas M, Mousa SS, Mousa SA. Comparative effectiveness of aflibercept for the treatment of patients with neovascular age-related macular degeneration. Clin Ophthalmol. 2013;7:495-501.

47. Sharma T, Dhingra R, Singh S, et al. Aflibercept: a novel VEGF targeted agent to explore the future perspectives of anti-angiogenic therapy for the treatment of multiple tumors. Mini Rev Med Chem. 2013;13: 530-540.

48. Chu QS. Aflibercept (AVE0005): an alternative strategy for inhibiting tumour angiogenesis by vascular endothelial growth factors. Expert Opin Biol Ther. 2009;9:263-271.

49. Roy H, Bhardwaj S, Babu M, et al. Adenovirus-mediated gene transfer of placental growth factor to perivascular tissue induces angiogenesis via upregulation of the expression of endogenous vascular endothelial growth factor-A. Hum Gene Ther. 2005;16:1422-1428.

50. Fischer C, Mazzone M, Jonckx B, Carmeliet P. FLT1 and its ligands VEGFB and PIGF: drug targets for anti-angiogenic therapy? Nat Rev Cancer. 2008;8:942-956.

51. Rini BI, Michaelson MD, Rosenberg JE, et al. Antitumor activity and biomarker analysis of sunitinib in patients with bevacizumab-refractory metastatic renal cell carcinoma. J Clin Oncol. 2008;26:3743-3748.

52. Gaya A, Tse V. A preclinical and clinical review of aflibercept for the management of cancer. Cancer Treat Rev. 2012;38:484-493.

53. Fetterly GJ, Aras U, Lal D, Murphy M, Meholick PD, Wang ES. Development of a preclinical PK/PD model to assess antitumor response of a sequential aflibercept and doxorubicin-dosing strategy in acute myeloid leukemia. AAPS J. 2013;15(3):662-673.

54. Van Cutsem E, Tabernero J, Lakomy R, et al. Addition of aflibercept to fluorouracil, leucovorin, and irinotecan improves survival in a phase III randomized trial in patients with metastatic colorectal cancer previously treated with an oxaliplatin-based regimen. J Clin Oncol. 2012;30: 3499-3506.

55. Sun W. Angiogenesis in metastatic colorectal cancer and the benefits of targeted therapy. J Hematol Oncol. 2012;5:63.

56. Bennouna J, Sastre J, Arnold D, et al; ML18147 Study Investigators. Continuation of bevacizumab after first progression in metastatic colorectal cancer (ML18147): a randomised phase 3 trial. Lancet Oncol. 2013;14:29-37.

57. Bou-Assaly W, Mukherji S. Cetuximab (erbitux). AJNR Am J Neuroradiol. 2010;31:626-627.

58. Vincenzi B, Zoccoli A, Pantano F, Venditti O, Galluzzo S. Cetuximab: from bench to bedside. Curr Cancer Drug Targets. 2010;10:80-95.

59. Khoukaz T. Administration of anti-EGFR therapy: a practical review. Semin Oncol Nurs. 2006;22:20-27.

60. Tortora G, Caputo R, Pomatico G, et al. Cooperative inhibitory effect of novel mixed backbone oligonucleotide targeting protein kinase $\mathrm{A}$ in combination with docetaxel and anti-epidermal growth factorreceptor antibody on human breast cancer cell growth. Clin Cancer Res. 1999;5:875-881. 
61. Mandal M, Adam L, Mendelsohn J, Kumar R. Nuclear targeting of Bax during apoptosis in human colorectal cancer cells. Oncogene. 1998;17:999-1007.

62. Kurai J, Chikumi H, Hashimoto K, et al. Antibody-dependent cellular cytotoxicity mediated by cetuximab against lung cancer cell lines. Clin Cancer Res. 2007;13:1552-1561.

63. Kimura H, Sakai K, Arao T, Shimoyama T, Tamura T, Nishio K. Antibody-dependent cellular cytotoxicity of cetuximab against tumor cells with wild-type or mutant epidermal growth factor receptor. Cancer Sci. 2007;98:1275-1280.

64. Bose D, Zimmerman LJ, Pierobon M, et al. Chemoresistant colorectal cancer cells and cancer stem cells mediate growth and survival of bystander cells. Br J Cancer. 2011;105:1759-1767.

65. Feng Y, Dai X, Li X, et al. EGF signalling pathway regulates colon cancer stem cell proliferation and apoptosis. Cell Prolif. 2012;45:413-419.

66. Chung KY, Shia J, Kemeny NE, et al. Cetuximab shows activity in colorectal cancer patients with tumors that do not express the epidermal growth factor receptor by immunohistochemistry. J Clin Oncol. 2005;23:1803-1810.

67. Jonker DJ, O'Callaghan CJ, Karapetis CS, et al. Cetuximab for the treatment of colorectal cancer. N Engl J Med. 2007;357:2040-2048.

68. Sobrero AF, Maurel J, Fehrenbacher L, et al. EPIC: phase III trial of cetuximab plus irinotecan after fluoropyrimidine and oxaliplatin failure in patients with metastatic colorectal cancer. J Clin Oncol. 2008;26: 2311-2319.

69. Borner M, Koeberle D, Von Moos R, et al; Swiss Group for Clinical Cancer Research (SAKK), Bern, Switzerland. Adding cetuximab to capecitabine plus oxaliplatin (XELOX) in first-line treatment of metastatic colorectal cancer: a randomized phase II trial of the Swiss Group for Clinical Cancer Research SAKK. Ann Oncol. 2008;19: $1288-1292$

70. Cunningham D, Humblet Y, Siena S, et al. Cetuximab monotherapy and cetuximab plus irinotecan in irinotecan-refractory metastatic colorectal cancer. N Engl J Med. 2004;351:337-345.

71. Cersosimo RJ. Management of advanced colorectal cancer, part 1. Am J Health Syst Pharm. 2013;70:395-406.

72. Cersosimo RJ. Management of advanced colorectal cancer, part 2. Am J Health Syst Pharm. 2013;70:491-506.

73. Hubbard JM, Alberts SR. Alternate dosing of cetuximab for patients with metastatic colorectal cancer. Gastrointest Cancer Res. 2013;6:47-55.

74. Bokemeyer C, Van Cutsem E, Rougier P, et al. Addition of cetuximab to chemotherapy as first-line treatment for KRAS wild-type metastatic colorectal cancer: pooled analysis of the CRYSTAL and OPUS randomised clinical trials. Eur J Cancer. 2012;48:1466-1475.

75. Tejpar S, Celik I, Schlichting M, Sartorius U, Bokemeyer C, Van Cutsem E. Association of KRAS G13D tumor mutations with outcome in patients with metastatic colorectal cancer treated with first-line chemotherapy with or without cetuximab. J Clin Oncol. 2012;30: 3570-3577.
76. Brand TM, Iida M, Wheeler DL. Molecular mechanisms of resistance to the EGFR monoclonal antibody cetuximab. Cancer Biol Ther. 2011;11: 777-792.

77. Kopper L. Panitumumab: an arrow on target. Pathol Oncol Res. 2010;16: 143-148.

78. Cartenì G, Fiorentino R, Vecchione L, Chiurazzi B, Battista C. Panitumumab a novel drug in cancer treatment. Ann Oncol. 2007; 18 Suppl 6:vi16-vi21.

79. Vectibix ${ }^{\circledR}$ (panitumumab) [package insert]. Thousand Oaks, CA: Amgen Inc; 2010.

80. Agency. EM. Vectibix European Public Assessment Report, Summary of Product Characteristics. 2010. Available from: http://wwwemaeuropaeu/ docs/en_GB/document_library/EPAR_-_Product_Information/ human/000741/WC500047710pdf. Accessed August 15, 2013.

81. Douillard JY, Siena S, Cassidy J, et al. Randomized, phase III trial of panitumumab with infusional fluorouracil, leucovorin, and oxaliplatin (FOLFOX4) versus FOLFOX4 alone as first-line treatment in patients with previously untreated metastatic colorectal cancer: the PRIME study. J Clin Oncol. 2010;28:4697-4705.

82. Peeters M, Price TJ, Cervantes A, et al. Randomized phase III study of panitumumab with fluorouracil, leucovorin, and irinotecan (FOLFIRI) compared with FOLFIRI alone as second-line treatment in patients with metastatic colorectal cancer. J Clin Oncol. 2010;28:4706-4713.

83. Cohn AL, Shumaker GC, Khandelwal P, et al. An open-label, singlearm, phase 2 trial of panitumumab plus FOLFIRI as second-line therapy in patients with metastatic colorectal cancer. Clin Colorectal Cancer. 2011;10:171-177.

84. Kohne CH, Hofheinz R, Mineur L, et al. First-line panitumumab plus irinotecan/5-fluorouracil/leucovorin treatment in patients with metastatic colorectal cancer. J Cancer Res Clin Oncol. 2012;138:65-72.

85. Wilhelm SM, Dumas J, Adnane L, et al. Regorafenib (BAY 73-4506): a new oral multikinase inhibitor of angiogenic, stromal and oncogenic receptor tyrosine kinases with potent preclinical antitumor activity. Int J Cancer. 2011;129:245-255.

86. Aprile G, Macerelli M, Giuliani F. Regorafenib for gastrointestinal malignancies : from preclinical data to clinical results of a novel multitarget inhibitor. BioDrugs. 2013;27:213-224.

87. Abou-Elkacem L, Arns S, Brix G, et al. Regorafenib inhibits growth, angiogenesis and metastasis in a highly aggressive, orthotopic colon cancer model. Mol Cancer Ther. 2013;12(7):1322-1331.

88. Schultheis B, Folprecht G, Kuhlmann J, et al. Regorafenib in combination with FOLFOX or FOLFIRI as first- or second-line treatment of colorectal cancer: results of a multicenter, phase Ib study. Ann Oncol. 2013;24:1560-1567.

89. George S, Wang Q, Heinrich MC, et al. Efficacy and safety of regorafenib in patients with metastatic and/or unresectable GI stromal tumor after failure of imatinib and sunitinib: a multicenter phase II trial. J Clin Oncol. 2012;30:2401-2407.
Drug Design, Development and Therapy

\section{Publish your work in this journal}

Drug Design, Development and Therapy is an international, peerreviewed open-access journal that spans the spectrum of drug design and development through to clinical applications. Clinical outcomes, patient safety, and programs for the development and effective, safe, and sustained use of medicines are a feature of the journal, which
Dovepress

has also been accepted for indexing on PubMed Central. The manuscript management system is completely online and includes a very quick and fair peer-review system, which is all easy to use. Visit http://www.dovepress.com/testimonials.php to read real quotes from published authors. 